\title{
Confocal laser scanning microscopy as a new valuable tool in the diagnosis of onychomycosis - comparison of six diagnostic methods
}

\author{
G. Rothmund, ${ }^{1 *}$ E. C. Sattler, ${ }^{2 *}$ R. Kaestle, ${ }^{1}$ C. Fischer, ${ }^{1}$ C. J. Haas, ${ }^{3}$ H. Starz ${ }^{1}$ and J. Welzel ${ }^{1}$ \\ ${ }^{1}$ Department of Dermatology and Allergology, General Hospital Augsburg, Augsburg, Germany, ${ }^{2}$ Department of Dermatology and Allergology, Ludwig- \\ Maximilians-University of Munich, Munich, Germany and ${ }^{3}$ Institute of Pathology, General Hospital Augsburg, Augsburg, Germany
}

\section{Summary}

\begin{abstract}
Onychomycosis is common and can mimic several different nail disorders. Accurate diagnosis is essential to choose the optimum antifungal therapy. The aim of this study was to evaluate the use of confocal laser scanning microscopy (CLSM) and optical coherence tomography (OCT) as new non-invasive diagnostic tools in onychomycosis and to compare them with the established techniques. In a prospective trial, 50 patients with suspected onychomycosis and 10 controls were examined by CLSM and OCT. Parallel KOH preparation, culture, PAS-staining and PCR were performed. PCR showed the highest sensitivity, followed by CLSM, PAS and KOH preparation. OCT offered the second best sensitivity but displayed the lowest specificity. CLSM and KOH preparation showed a high specificity and CLSM offered the best positive predictive value, similar to $\mathrm{KOH}$ preparation and OCT. Fungal culture showed the lowest sensitivity and the worst negative predictive value, yet culture and PCR are the only techniques able to identify genus and species. In summary, CLSM was comparable to PAS staining and superior to KOH preparation. Due to the low specificity we assess OCT not as appropriate. In the differentiation of species PCR outplays the fungal culture in terms of time and sensitivity.
\end{abstract}

Key words: confocal laser scanning microscopy, PAS staining, fungal culture, onychomycosis, KOH preparation, Optical coherence tomography.

\section{Background}

Nail diseases are often very troublesome to the patient $^{1,2}$ and can be diagnostically challenging even to the experienced dermatologist. ${ }^{3-5}$ Especially, onychomycosis is a common problem ${ }^{6-8}$ and can sometimes be difficult to be distinguished from other nail disorders like psoriasis, lichen ruber or eczematous nails. ${ }^{9,10}$ The development of optimised antimycotic

Correspondence: J. Welzel, Head of the Department of Dermatology and Allergology, General Hospital Augsburg, Sauerbruchstraße 6, 86179 Augsburg, Germany.

Tel.: +49821400 7401. Fax: +4982140017 7401.

E-mail: julia.welzel@klinikum-augsburg.de

*Both authors contributed equally. therapy regimens stresses the need for accurate diagnosis and species differentiation, especially in therapy refractant and recurrent disease. ${ }^{11-17}$ Hitherto existing diagnostic methods include $\mathrm{KOH}$ preparation, dermatophyte culture, PCR, and histopathology with PASstaining, ${ }^{18}$ but they all share certain drawbacks in terms of sensitivity or specificity, time delay, or costintensity and effort. ${ }^{19-23}$

Confocal laser scanning microscopy (CLSM) and optical coherence tomography (OCT) offer a quick and non-invasive view of the patients nail in real-time. So far, morphological features of the healthy nail plate in CLSM were recently described and compared with typical findings in leuconychia and onychomycosis. ${ }^{24}$ In a few other reports, the possibility of diagnosing onychomycosis by CLSM and OCT was demonstrated. $^{25-27}$ Also, recently, digital dermoscopy of the nail was shown to be helpful in the diagnosis of onychomycosis, as distinctive dermoscopic signs were 
found that were exclusively seen in distal subungual onychomycosis. $^{28}$ The aim of this prospective study was the evaluation of CLSM and OCT as diagnostic tool in onychomycosis in terms of sensitivity, specificity, positive and negative predictive value and practicability in comparison with the hitherto established diagnostic methods.

\section{Patients and methods}

\section{Patients}

Sixty patients (aged 11-91 years, 40 male, 20 female) with nail disorders were included in this prospective study. There were no limitations in age or gender. Also, predisposing factors for the development of onychomycosis and possible co-factors were not taken into account. Within this group, 50 patients were clinically suspicious for onychomycosis and 10 patients had nail diseases of other origin.

\section{Onychomycosis group}

Fifty patients with the clinical typical picture of onychomycosis were recruited. Although there were no limitations to the location, only onychomycosis of the toe nails were seen. According to common classifications ${ }^{29-31}, 27$ patients $(54 \%)$ showed a distolateral subungual onychomycosis, 17 cases (34\%) a total dystrophic onychomycosis, five patients (10\%) appeared as superficial white onychomycosis and only one patient displayed the picture of a proximal subungual onychomycosis. There were no limitations to age or gender, displaying an age-range from 39 to 91 years (mean of 74.4 years) and a gender distribution of 33 male and 17 female patients.

\section{Control group}

As controls served a collective of 10 panellists (seven males and three females), aged 11-91 (mean age of 50.4 years) with nail disorders not typical for onychomycosis like oil spots, motteling or longitudinal creases and with additional skin disorders, commonly associated with nail deviations (six patients with psoriasis, two with eczema, one with lichen ruber planus and one with a nail destruction following chemotherapy).

Exclusion criteria for both groups were the prior externally carried out diagnosis of onychomycosis and antimycotic therapy within the 3 month prior to recruitment.

\section{Methods}

a. KOH-preparation: The sampled material was incubated with a $15 \%$ caustic potash solution for $15 \mathrm{~min}$ and then scanned by light microscopy.

b. Fungal culture: Suspicious material was placed on a selective agar for pathogenic fungi, and evaluation and differentiation of species were carried out after up to 4 weeks of incubation.

c. PCR: Verification of the presence of dermatophytes was carried out with a combination of two techniques: First, the $184 \mathrm{bp}$ segment from the high variable domaine of the small ribosomal subunit of the 18S-rRNA gene was amplified with the primer DH1L and DH1R. This was followed by a restrictionfragment-length-polymorphism analysis (RFLP) with EaeI $(=\mathrm{Cfrl})$ as restriction enzyme, which specifically in dermatophytes splits the $184 \mathrm{bp}$ PCR product in a $130 \mathrm{bp}$ and a 54 bp fragment. ${ }^{32,33}$

Secondly, for the detection of dermatophytes, a pandermatophyte PCR was conducted with panDER-1 and panDER-2 as primers, which detect the fragments that encode the chitin-synthase 1 in dermatophytes leading to amplification products with a length of $366 \mathrm{bp} .^{34}$

Specification of the detected dermatophytes was performed via PCR of the internal transcribed spacer gene 1 (ITS1) of Trichophyton rubrum (Trub-1, Trub-2) and of the ITS1 of Trichophyton mentagrophytes (TmentF-, Tment-R). Quality of the isolated DNA was checked for by a multiplex-PCR.

The product of the PCR for the detection of Microsporum canis is the $211 \mathrm{bp}$-segment from the DNA of the Topoisomerase II of the dermatophytes. ${ }^{35}$

DNA isolation was conducted with the DNeasy Blood \& Tissue Kit (\#69506, Qiagen, Hilden, Germany), Amplitaq-Gold (\#N8080259, Applied Biosystems, Darmstadt, Germany) was used as DNA polymerase. Annealing temperature was $60{ }^{\circ} \mathrm{C}$.

d. Histopathology with PAS staining: Material was embedded in paraffin and stained with thePASreaction.

e. CLSM was performed with the VivaScope 1500 Multiwave (Fa. MAVIG GmbH, Munich, Germany) with the $830 \mathrm{~nm}$ laser in the reflection mode. Horizontal levels, so called VivaStacks, in different depths were scanned down to the nail bed. The single image is $500 \times 500 \mu \mathrm{m}$ in size. The clinically suspicious zone of the nail was scanned and white lengthy or thready structures with high reflection and typical shape or spore-like bright aggregates similar to their shape seen in histology were counted as positive for fungal infection. 
f. Optical coherence tomography was performed using the SD-OCT-System Callisto (Fa. Thorlabs HL AG, Lübeck, Germany), offering a lateral resolution of $15 \mu \mathrm{m}$ and an axial resolution of $7 \mu \mathrm{m}$. The penetration depth lies about $1 \mathrm{~mm}$ limited due to scattering. In contrast to CLSM, the resulting images are vertical slices with a length of 4-6 mm. Again, white lengthy or thready structures with high reflection or round aggregated white structures were valued as fungal structures.

\section{Statistical analysis}

Sensitivity and specificity were calculated for each diagnostic method. Sensitivity as truly positive rate in our study showed the percentage in which the technique was able to detect the fungal infection within all patients with onychomycosis. Specificity, in contrast as the truly negative rate, gave the fraction of patients that were correctly found to have no onychomycosis.

The positive predictive value (PPV) describes the probability that a positive result really relies on a positive finding (in our case really an onychomycosis), so it is offering the fraction of truly positive found results from all positive results. Accordingly, the negative predictive value (NPV) answers the question in which percentage a negative result really relies on a negative finding, so in our case, in how many negative results out of all negative results the patients really had no onychomycosis.

As basis for calculations fungal culture, PCR and histopathology (PAS staining) were defined as gold standard, so that a positive finding in either one of the three confirmed the diagnosis of onychomycosis.

\section{Results}

Among the 60 patients of this study, the diagnosis of onychomycosis was established in 65\% (39 patients), meaning that at least one of the three as gold standarddefined methods (PCR, fungal culture, PAS staining) showed a positive result in two-thirds of all 60 cases. Of these 39 patients, 36 patients $(92 \%)$ belonged to the onychomycosis group and 3 patients $(8 \%)$ to the control group. Two-thirds of these patients were male (26/39) and one-third female (13/39) displaying the same gender ratio as the entire collective also with two-thirds being male (40/60). Age ranged from 11 to 91 years.

\section{Sensitivity, specificity, PPV and NPV}

The positive results over all for each technique and sensitivity, specificity, PPV and NPV for all six diagnostic methods are given in Table 1.

\section{Sensitivity}

Polymerase chain reaction offered the best sensitivity with $94.9 \%$, followed by OCT (92.3\%), CLSM (79.5\%), $\mathrm{KOH}$-preparation (74.4\%), histopathology (PAS-staining) $(69.2 \%)$ and far of last the fungal culture with $20.5 \%$. A comparison of the sensitivity of all diagnostic methods for onychomycosis tested is shown in Fig. 1.

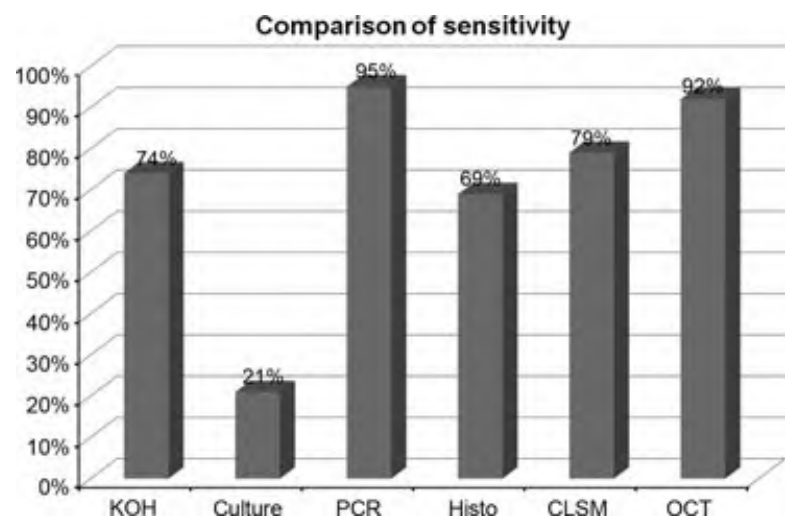

Figure 1 Comparison of sensitivity of all six diagnostic methods.
Table 1 Overview of all results for all six diagnostic methods in terms of overall positive results, sensitivity, specificity and positive and negative predictive value.

\begin{tabular}{llllll}
\hline $\begin{array}{l}\text { Diagnostic } \\
\text { method }\end{array}$ & $\begin{array}{l}\text { Positive } \\
\text { results (\%) }\end{array}$ & $\begin{array}{l}\text { Sensitivity } \\
(\%)\end{array}$ & $\begin{array}{l}\text { Specificity } \\
(\%)\end{array}$ & $\begin{array}{l}\text { Positive } \\
\text { predictive } \\
\text { value (\%) }\end{array}$ & $\begin{array}{l}\text { Negative } \\
\text { predictive } \\
\text { value (\%) }\end{array}$ \\
\hline KOH-preparation & 56.7 & 74.4 & 76.2 & 85.3 & 61.5 \\
Fungal culture & 13.3 & 20.5 & $100^{*}$ & $100^{*}$ & 40.4 \\
PCR & 61.7 & 94.9 & $100^{*}$ & $100^{*}$ & 91.3 \\
PAS staining & 45 & 69.2 & $100^{*}$ & $100^{*}$ & 63.6 \\
CLSM & 58.3 & 79.5 & 81 & 88.6 & 85.3 \\
OCT & 80 & 92.3 & 42.9 & 75 & 75 \\
\hline
\end{tabular}

*These three methods were defined as gold standards in the study design therefore being $100 \%$ in specificity and PPV. 


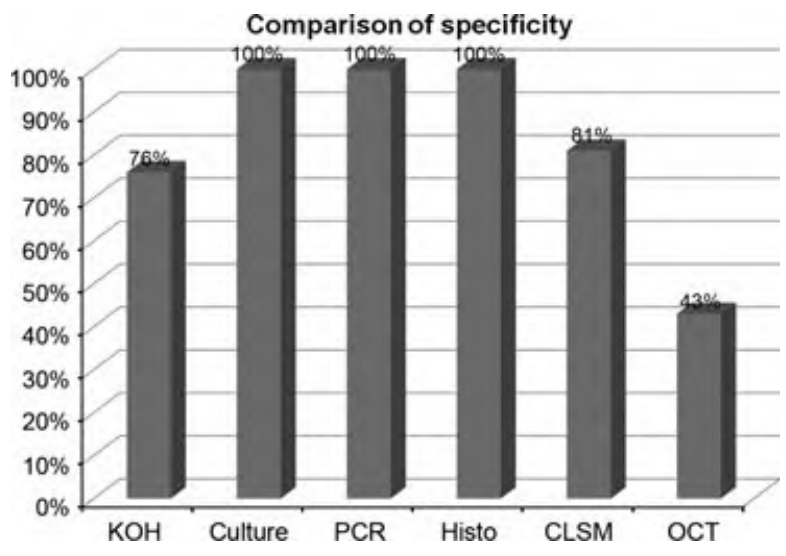

Figure 2 Comparison of the specificity of all six diagnostic methods.

\section{Specificity}

Due to the study design PCR, culture and histopathology were defined as gold standard, therefore stating a specificity of $100 \%$. Compared with these gold standard techniques, CLSM showed the best value for specificity with $81 \%$, followed by $\mathrm{KOH}$-preparation with $76.2 \%$ and far off last the OCT with only $42.9 \%$. The comparison for all six techniques is graphically demonstrated in Fig. 2.

For the non-invasive optical methods, CLSM and OCT, results are given in detail below, allowing also a comparison with the gold standard techniques as well as a comparison in terms of positive and negative correlation between the six different methods conducted.

\section{CLSM}

Confocal laser scanning microscopy showed a positive result in 58.3\% (35/60), similar to the $\mathrm{KOH}$-preparation (56.7\%) and slightly above average (52.5\%), pertaining to all methods. Two exemplary pictures are given in Fig. 3a and b. Of the positive results, 97.1\% were found in the onychomycosis group and $2.9 \%$ in the control group.

Sensitivity of CLSM was high with 79.5\%, comparable to that of $\mathrm{KOH}$-preparation (74.4\%), and also specificity was high with $81 \%$. Due to the high number of truly positive results, the PPV is high with $88.6 \%$, also similar to that of $\mathrm{KOH}$-preparation with $85.3 \%$. The NPV with $68 \%$ is slightly above average (66.6\%).

Compared with the gold standard techniques, CLSM offers a much higher sensitivity with $79.5 \%$ than the culture $(20.5 \%)$ and than histopathology (69.2\%), yet PCR tops all with $94.9 \%$. The NPV of CLSM behaves similarly: with $68 \%$ better than culture $(40.4 \%)$ and histopathology (63.6\%), but clearly under the $91.3 \%$ of the PCR. In addition, CLSM shows - except for the defined gold standards - the best specificity with $81 \%$ and the best PPV with $88.6 \%$.

Correlating CLSM with the other methods (pertaining to the entire collective), the best positive accordance is seen with the OCT in $56.7 \%$, the worst with culture in only $13.3 \%$. With the negative correlation, it is vice versa: only $18.3 \%$ compared to OCT and $41.7 \%$ compared to culture (Fig. 4).

\section{OCT}

With $80 \%$ (48/60), overall positive results the OCT ranked far over the average of $52.5 \%$. This highest number of all positive results compared with all other techniques was found in $91.7 \%(44 / 48)$ in the onychomycosis group and in $8.3 \%(4 / 48)$ in the control group. A typical positively rated OCT image is shown in Fig. 5.

Due to the high number of positive results, OCT shows a high sensitivity of $92.3 \%$, right behind the PCR with $94.9 \%$, but due to the very low number of truly
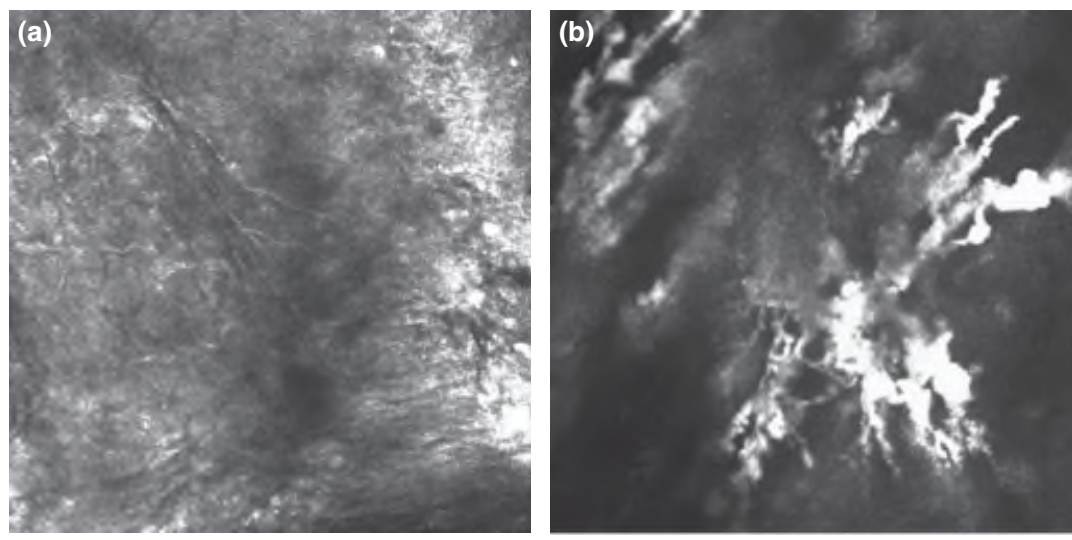

Figure 3 (a) Typical features of dermatophytes within the nail plate in CLSM with lengthy structures with high reflection and typical shape of hyphae. (b) In addition to the thready like structures with high reflection already shown in Fig. 3a in this confocal lasermicroscopic image of the nail plate, spore like aggregates with high reflection similar to their appearance in histopathology are visible. 


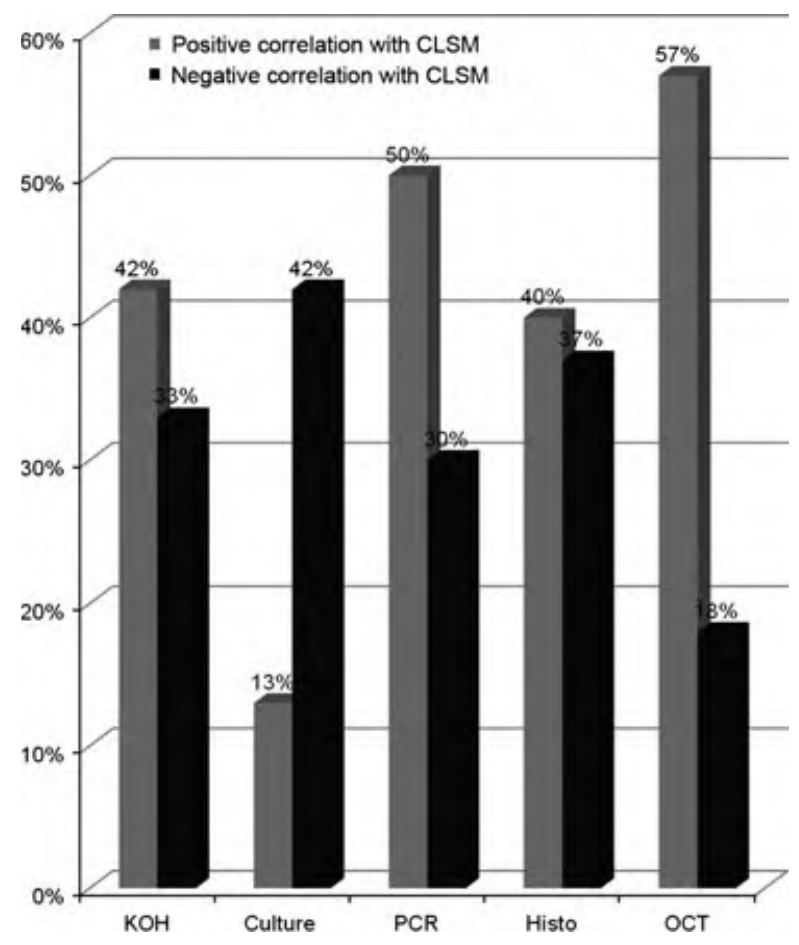

Figure 4 Correlation of the results of CLSM with all other diagnostic methods.

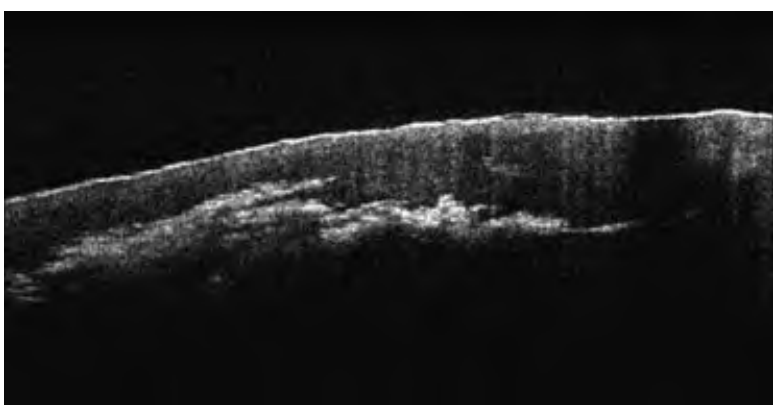

Figure 5 Typical features of onychomycosis in OCT with lengthy structures with high reflection.

negative results, the specificity of $42.9 \%$ was poor and the worst compared with all other techniques. The PPV is also ranked last with $75 \%$, the NPV with $75 \%$ is above average and again second best after the PCR (91.3\%).

Compared with the gold standards, the sensitivity of OCT reaches with $92.3 \%$ almost the results of the PCR (94.9\%) and is much better than the culture $(20.9 \%)$ and also than the histopathology (69.2\%). The NPV of OCT lies with $75 \%$ also above the $63.6 \%$ of histopathology and clearly above the $40.4 \%$ of the culture, but is also markedly below that of the PCR (91.3\%).

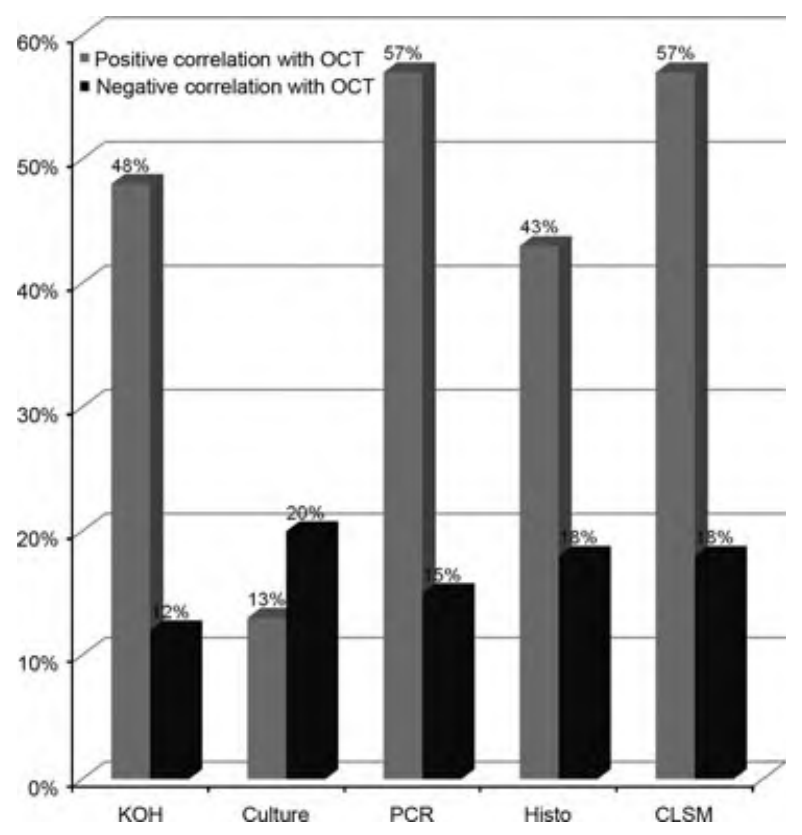

Figure 6 Correlation of the results of OCT with all other diagnostic methods.

Correlation of OCT with the other methods, pertaining to all 60 patients, showed a good positive accordance with the PCR and the CLSM each with $56.7 \%$. The worst positive correlation was seen with the fungal culture in only $13.3 \%$. Negative accordance of OCT lay only between $11.7 \%$ compared with $\mathrm{KOH}-$ preparation and a maximum of $20 \%$ compared with culture (Fig. 6).

\section{Differentiation of species}

The only two methods allowing a differentiation of species are the PCR and the fungal culture. In both techniques, only Trichophyton rubrum and Tr. mentagrophytes were found.

The PCR found Trichophyton rubrum in $81.1 \%$ (30/37) of all positive PCR results and Trichophyton mentagrophytes in $18.9 \%(7 / 37)$. In the fungal culture, which was only positive in $13.3 \%$, the growth of $\mathrm{Tr}$. rubrum was seen in $87.5 \%(7 / 8)$ and Tr. mentagrophytes grew in $12.5 \%(1 / 8)$.

\section{Time frame, costs, efforts}

A rough estimation of all six diagnostic methods of the time frame until diagnosis can be confirmed, of the costs per examination (according to the German medical fee schedule GOÄ), of the acquisition cost of the devices plus consumables plus experienced personnel needed, as well 
as the ability to differentiate between species, is shown in Table 2.

\section{Discussion}

All evaluations of the laboratory diagnostic methods (KOH, culture, PCR and PAS) presuppose the correct sampling of the material. Especially, the correct prior disinfection, knowledge on the best area of the nail for sampling and a sufficient amount of material obtained are essential to avoid false negative results and contamination. ${ }^{5,26}$ For each diagnostic method, possible disturbing factors, advantages and drawbacks and a comparison with results in the literature are discussed below.

\section{$\mathrm{KOH}$ preparation}

This easy to perform and, being a low budget method, offers a quick result after about $30 \mathrm{~min}$. It is very well suitable as a screening method and applicable in every doctor's office. Most important requirements are sufficient sampling and an experienced examiner. The good sensitivity of $74.4 \%$ we found is comparable to that of histopathology and compared with the literature, falls into the higher end of the range stated between 25\% and $80 \% .^{36,37}$ The specificity of $76.2 \%$ and the positive predictive value of $85.3 \%$ were also comparable to the $72 \%$ and $88 \%$ found by Weinberg et al. [37] However, the NPV of $61.5 \%$ was the second worst after the culture with $40.4 \%$, also in accordance with the literature stating a NPV of $58 \%$ for the $\mathrm{KOH}$-preparation and $43 \%$ for the culture, ${ }^{37}$ and $\mathrm{KOH}$ preparation is not able to differentiate species.

\section{Fungal culture}

In our study, sensitivity was poor with $20.5 \%$, lying even below the lowest data given in the literature with a range between $23 \%$ and $80 \%$, with most studies narrowing down between $32 \%$ and $59 \% .^{37,38}$ As sampling was the same for all methods, possible explanations for the poor sensitivity in our study could be a low number of germs or impaired vitality, but this remains uncertain. Due to our study design, specificity was nominated as $100 \%$. Weinberg et al. [37] found it at $82 \%$. Due to the high number of false negative results, the NPV is poor with $40.4 \%$ similar to $43 \%$ described previously combined with a good PPV of $90 \%,{ }^{37}$ which was $100 \%$ in our results as the culture was defined as gold standard.

Yet, of all methods, only the fungal culture and the PCR are able to differentiate the species to allow for an appropriate antimycotic treatment, and fungal culture is the only method to proof vitality of the pathogens, which is important in therapy refractory and recurrent disease. Fungal culture is about five times cheaper than the PCR (see Table 2), but it takes 3-4 weeks until results are present compared to 1-2 days with the PCR, where the actual reaction only takes a few hours. ${ }^{34}$

\section{PCR}

Results of the PCR were superior to all other methods, except in terms of costs. PCR showed the best sensitivity with $94.9 \%$ and the highest NPV with $91.4 \%$, a little higher than the data seen by Kondori et al. [39] 2010 with $85 \%$ and $87 \%$ respectively, who combined a PanDermatophytes PCR with a Tr. rubrum-specific PCR. They found the specificity of this combination to be $94 \%$ with a PPV of $93 \% .^{39}$ As the PCR was defined as gold standard, our specificity and PPV were set at $100 \%$. Similar results were also found by Li et al. [40] using a triplex PCR.

A big advantage next to the fast results stated above and the possibility of germ differentiation is the little amount of pathogens needed (10-100 germs per ml are sufficient) for. ${ }^{40}$ This is also independent of the vitality of the fungi, which could be impaired if prior antimycotic therapy was conducted. The price for this quick

Table 2 Comparison of all six diagnostic methods in terms of time frame until receiving final results, costs per examination (according to the German medical fee schedule GOÄ), costs of the acquisition of the device, the consumables and the personnel and laboratory effort needed and a comparison of the ability to differentiate between different genus and species of the fungi.

\begin{tabular}{|c|c|c|c|c|}
\hline $\begin{array}{l}\text { Diagnostic } \\
\text { method }\end{array}$ & $\begin{array}{l}\text { Time frame } \\
\text { until diagnosis }\end{array}$ & $\begin{array}{l}\text { Costs per } \\
\text { examination }(€)\end{array}$ & $\begin{array}{l}\text { Acquisition costs }+ \\
\text { material/personnel }\end{array}$ & $\begin{array}{l}\text { Differentiation } \\
\text { of species }\end{array}$ \\
\hline $\mathrm{KOH}$-preparation & ca. $30 \mathrm{~min}$ & 10.36 & Low & No \\
\hline Fungal culture & 3-4 weeks & 27 & Low & Possible \\
\hline PCR & $1-2$ days & 127 & $\begin{array}{l}\text { High (material + several } \\
\text { devices + personnel) }\end{array}$ & Possible \\
\hline PAS staining & $1-2$ days & 77 & High (lab + material + personnel) & No \\
\hline CLSM & ca. $20 \mathrm{~min}$ & 140 & High (device ca. 85 000) & No \\
\hline OCT & ca. $15 \mathrm{~min}$ & 80 & High (device ca. 20 000) & No \\
\hline
\end{tabular}


and accurate tool is higher in terms of costs of acquisition (devices needed: thermocycler, centrifuges, incubator, elektrophorese apparatus, UV-screen), and consumables (KNA-Kit, TAq-Polymerase, Gel Pipettes, tubes etc.), which make this technique not appropriate for use in an everyday practice. However, for the use in the clinic, it should be considered as gold standard, in any case in therapy refractory cases or in recurrent or severe disease. ${ }^{17}$

\section{Histopathology (PAS staining)}

With a sensitivity of $69.2 \%$, histopathology was right behind $\mathrm{KOH}$ preparation $(74.4 \%)$, but its NPV was a little better with $63.6 \%$ vs. $61.5 \%(\mathrm{KOH})$, but in both terms, much better than the fungal culture $(20.5 \%$ and $40.4 \%)$. Other studies found an even higher sensitivity for PAS staining between $85 \%$ and $92 \% .^{37,38}$ and a specificity of $72 \%$, a PPV of $89.7 \%$ and a NPV of $77 \%{ }^{37}$ Again, as one of the gold standard methods in our study, specificity and PPV were both $100 \%$ and the NPV was a little lower compared with Weinberg's data with only $63.6 \%$, showing still quite a high number of false negative results and stressing the importance of correct sampling of the material by nail clipping for this method. One advantage is the ability to see if the fungi are located subungual or if they grow invasively into the nail organ. ${ }^{31}$ Results are available after 1-2 days, but there is no information on vitality or species. ${ }^{31}$ The equipment needed reserves this technique for the clinic or larger labs. ${ }^{41}$ The price lies clearly above that of a $\mathrm{KOH}$ preparation and culture, but below the PCR and is similar to that of OCT (see Table. 2).

\section{CLSM}

While CLSM is well established as helpful non-invasive tool with almost histological resolution in the diagnosis of lesions of the human skin, primarily in pigmented lesions but also in other skin tumours and diseases; ${ }^{42-49}$ it can also be helpfully applied for the examination of the nail plate. In a recent study, morphological features of CLSM of the healthy nail plate were described and compared with the typical findings in onychomycosis and leuconychia. ${ }^{24}$ Already in 1994 and 2001, CLSM was described as a diagnostic tool of the future, ${ }^{50,51}$ and also a case report by Hongcharu et al. [25] showed that CLSM was able to confirm the diagnosis of onychomycosis and the advantages of CLSM with a possibly higher precision and faster results compared with $\mathrm{KOH}$ preparation and dermatophyte culture were discussed. Feuilhade de Chavin described CLSM as a good diag- nostic method, but assessed it to be too complicated and expensive for routine measurements. ${ }^{26}$ Arrese et al. [52] saw the advantage of CLSM in the ability to demonstrate location and density of the fungi, therefore being able to quantify the 'fungal load'. Gupta et al. [29] also assess CLSM as a quick and reliable method, but find fault with the inability to differentiate between dermatophytes and moulds and yeasts.

Confocal laser scanning microscopy showed the third best sensitivity with $79.5 \%$ and the best specificity and PPV with $81 \%$ and $88.6 \%$ respectively, superior to $\mathrm{KOH}$ preparation, culture and OCT. NPV was with $85.3 \%$, second best right after the PCR (91.3\%).

As an optical method, false negative results due to wrong material sampling can be excluded and the risk of injury of the patient can be omitted. Results are quick within 20 minutes and due to the nearly histological resolution, also small amounts of fungi can be detected. Drawbacks are high acquisition costs for the device of about 85 000-120 000 Euro plus consumables and the need of a highly experienced examiner esp. when imaging the nail plate. The challenging handling when measuring the convex hard surface of the nail plate might be relieved soon with a newly developed handheld device.

Although differentiation of species and vitality is not possible, CLSM achieves results superior to $\mathrm{KOH}$ preparation and histopathology. We therefore feel that CLSM offers a helpful amendment of the hitherto existing diagnostic armamentarium in fungal infections of the nail.

\section{OCT}

Although OCT showed the second highest sensitivity with $92.3 \%$, this forfeits its informative value due to a specificity of only $42.9 \%$. PPV and NPV were $75 \%$ each, verifying esp. a high number of false positive results. This is probably due to the lower resolution, which does not allow a clear-cut differentiation between hyphae/spores and other nail creases/artefacts like trapped air that might appear similarly.

Like CLSM, OCT is a quick and non-invasive optical method offering results in real-time, ${ }^{53-55}$ but acquisition costs of the device are about 20000 Euro and again an experienced examiner is needed. In a pilot study by Abuzahra et al., OCT was compared with $\mathrm{KOH}$ preparation, culture and histology. OCT was able to detect fungal elements in all histologically positive specimens. No false positive results were seen in their controls, ${ }^{56}$ in contrast to our findings of a very low specificity and a high number of false positive results. 
Optical coherence tomography has a high enough resolution for measuring the nail thickness and displaying the different layers of the nail organ and individual structures, ${ }^{57,58}$ but due to the low specificity and the not histological resolution, it is, from our point of view, not useful as a standard method in the diagnosis of onychomycosis.

\section{References}

1 Drake LA, Patrick DL, Fleckman P et al. The impact of onychomycosis on quality of life: development of an international onychomycosis-specific questionnaire to measure patient quality of life. J Am Acad Dermatol 1999; 41(2 Pt 1): 189-96.

2 Lubeck DP. Measuring health-related quality of life in onychomycosis. J Am Acad Dermatol 1998; 38 (5 Pt 3): S64-8.

3 Korting HC, Schaller M. [New developments in medical mycology]. Hautarzt 2001; 52: 91-7.

4 olde Hartman TC, van Rijswijk E. Fungal nail infection. BMJ 2008; 337: a429.

5 Seebacher C, Brasch J, Abeck D et al. Onychomycosis. Mycoses 2007: 50: 321-7.

6 Burzykowski T, Molenberghs G, Abeck D et al. High prevalence of foot diseases in Europe: results of the Achilles Project. Mycoses 2003; 12: 496-505.

7 Ghannoum MA, Hajjeh RA, Scher R et al. A large-scale North American study of fungal isolates from nails: the frequency of onychomycosis, fungal distribution, and antifungal susceptibility patterns. J Am Acad Dermatol 2000; 43: 641-8.

8 Kaur R, Kashyap B, Bhalla P. Onychomycosis - epidemiology, diagnosis and management. Indian J Med Microbiol 2008; 26 : 108-16.

9 Allevato MA. Diseases mimicking onychomycosis. Clin Dermatol 2010; 28: 164-77.

10 Oppel T, Korting HC. Onychodystrophy and its management. Ger Med Sci 2003; 1: Doc02.

11 Baran R, Sigurgeirsson B, de Berker D et al. A multicentre, randomized, controlled study of the efficacy, safety and costeffectiveness of a combination therapy with amorolfine nail lacquer and oral terbinafine compared with oral terbinafine alone for the treatment of onychomycosis with matrix involvement. Br I Dermatol 2007: 157: 149-57.

12 Epstein E. How often does oral treatment of toenail onychomycosis produce a disease-free nail? An analysis of published data. Arch Dermatol 1998; 134: 1551-4.

13 Haneke E. [Therapy of onychomycoses]. Hautarzt 1993; 44: 335-46.

14 Korting HC, Ollert M, Abeck D. Results of German multicenter study of antimicrobial susceptibilities of Trichophyton rubrum and Trichophyton mentagrophytes strains causing tinea unguium. German Collaborative Dermatophyte Drug Susceptibility Study Group. Antimicrob Agents Chemother 1995; 39: 1206-8.

15 Korting HC, Schollmann C. The significance of itraconazole for treatment of fungal infections of skin, nails and mucous membranes. J Dtsch Dermatol Ges 2009; 7: 11-9-20.

16 Scher RK, Tavakkol A, Sigurgeirsson B et al. Onychomycosis: diagnosis and definition of cure. J Am Acad Dermatol 2007; 56: 939-44.
17 Brasch J, Shimanovich I. Persistent fingernail onychomycosis caused by Fusarium proliferatum in a healthy woman. Mycoses 2012; 55: 86-9.

18 Beifuss B, Borelli C, Korting HC. [Mycological laboratory]. Hautarzt 2006; 57: 487-8.90-2.

19 [No authors listed]. Fungal nail infections: diagnosis and management. Prescrire Int 2009; 18: 26-30.

20 Gupta AK, Drummond-Main C, Cooper EA, Brintnell W, Piraccini BM, Tosti A. Systematic review of nondermatophyte mold onychomycosis: diagnosis, clinical types, epidemiology, and treatment. J Am Acad Dermatol 2012; 66: 494-502.

21 Welsh O, Vera-Cabrera L, Welsh E. Onychomycosis. Clin Dermatol 2010; 28: 151-9.

22 Nenoff P, Ginter-Hanselmayer G, Tietz HJ. [Fungal nail infections - an update : part 2 - From the causative agent to diagnosis - conventional and molecular procedures.]. Hautarzt 2012; 63: 30-8.

23 Nenoff P, Ginter-Hanselmayer G, Tietz HJ. [Fungal nail infections - an Update : part 1 - Prevalence, epidemiology, predisposing conditions, and differential diagnosis.]. Hautarzt 2012; 63: 130-7.

24 Sattler E, Kaestle R, Rothmund G, Welzel J. Confocal laser microscopy, optical coherence tomography and transonychial water loss for in vivo investigation of nails. $\mathrm{Br} J$ Dermatol 2012; 166: 740-6.

25 Hongcharu W, Dwyer P, Gonzalez S, Anderson RR. Confirmation of onychomycosis by in vivo confocal microscopy. J Am Acad Dermatol 2000; 42(2 Pt 1): 214-6.

26 Feuilhade de Chauvin M. New diagnostic techniques. J Eur Acad Dermatol Venereol 2005; 19(Suppl 1): 20-4.

27 Markus R, Huzaira M, Anderson RR, Gonzalez S. A better potassium hydroxide preparation? In vivo diagnosis of tinea with confocal microscopy. Arch Dermatol 2001; 137: 1076-8.

28 Piraccini BM, Balestri R, Starace M, Rech G. Nail digital dermoscopy (Onychoscopy) in the diagnosis of onychomycosis. J Eur Acad Dermatol Venereol 2011; doi: 10.1111/j.14683083.2011.04323.x.

29 Gupta AK, Ryder JE, Summerbell RC. Onychomycosis: classification and diagnosis. J Drugs Dermatol 2004; 3: 51-6.

30 Hay RJ, Baran R. Onychomycosis: a proposed revision of the clinical classification. J Am Acad Dermatol 2011; 65: 1219 27.

31 Reinel D. Onychomykose [Onychomycosis]. Hautarzt 2004; 55: 143-9.

32 Gutzmer R, Mommert S, Kuttler U, Werfel T, Kapp A. Rapid identification and differentiation of fungal DNA in dermatological specimens by LightCycler PCR. J Med Microbiol 2004; 53(Pt 12): 1207-14.

33 Machouart-Dubach M, Lacroix C, de C et al. Rapid discrimination among dermatophytes, Scytalidium spp., and other fungi with a PCR-restriction fragment length polymorphism ribotyping method. J Clin Microbiol 2001; 39: 685-90.

34 Brillowska-Dabrowska A, Saunte DM, Arendrup MC. Fivehour diagnosis of dermatophyte nail infections with specific detection of Trichophyton rubrum. J Clin Microbiol 2007; 45: 1200-4.

35 Kanbe T, Suzuki Y, Kamiya A et al. Species-identification of dermatophytes Trichophyton, Microsporum and Epidermophyton by PCR and PCR-RFLP targeting of the DNA topoisomerase II genes. J Dermatol Sci 2003; 33: 41-54. 
36 Reisberger EM, Abels C, Landthaler M, Szeimies RM. Histopathological diagnosis of onychomycosis by periodic acidSchiff-stained nail clippings. Br J Dermatol 2003; 148: 74954.

37 Weinberg JM, Koestenblatt EK, Tutrone WD, Tishler HR, Najarian L. Comparison of diagnostic methods in the evaluation of onychomycosis. J Am Acad Dermatol 2003; 49: 193-7.

38 Lawry MA, Haneke E, Strobeck K, Martin S, Zimmer B, Romano PS. Methods for diagnosing onychomycosis: a comparative study and review of the literature. Arch Dermatol 2000; 136: 1112-6.

39 Kondori N, Abrahamsson AL, Ataollahy N, Wenneras C. Comparison of a new commercial test, Dermatophyte-PCR kit, with conventional methods for rapid detection and identification of Trichophyton rubrum in nail specimens. Med Mycol 2010; 48: 1005-8.

40 Li XF, Tian W, Wang H et al. Direct detection and differentiation of causative fungi of onychomycosis by multiplex polymerase chain reaction-based assay. Eur J Dermatol 2011; 21 : $37-42$.

41 Richert B, Lateur N, Theunis A, Andre J. New tools in nail disorders. Semin Cutan Med Surg 2009; 28: 44-8.

42 Astner S, Ulrich M. [Confocal laser scanning microscopy]. Hautarzt 2010; 61: 421-8.

43 Gonzalez S, Gilaberte-Calzada Y. In vivo reflectance-mode confocal microscopy in clinical dermatology and cosmetology. Int J Cosmet Sci 2008; 30: 1-17.

44 Pellacani G, Longo C, Malvehy J et al. In vivo confocal microscopic and histopathologic correlations of dermoscopic features in 202 melanocytic lesions. Arch Dermatol 2008; 144: 1597-608.

45 Pellacani G, Scope A, Ferrari B et al. New insights into nevogenesis: in vivo characterization and follow-up of melanocytic nevi by reflectance confocal microscopy. J Am Acad Dermatol 2009; 61: 1001-13.

46 Pellacani G, Vinceti M, Bassoli S et al. Reflectance confocal microscopy and features of melanocytic lesions: an internet-based study of the reproducibility of terminology. Arch Dermatol 2009; 145: 1137-43.
47 Ulrich M, Stockfleth E, Roewert-Huber J, Astner S. Noninvasive diagnostic tools for nonmelanoma skin cancer. $\mathrm{Br} J \mathrm{Der}$ matol 2007; 157(Suppl 2): 56-8.

48 Astner S, Gonzalez S, Cuevas J et al. Preliminary evaluation of benign vascular lesions using in vivo reflectance confocal microscopy. Dermatol Surg 2010; 36: 1099-110.

49 Hegyi J, Hegyi V, Messer G, Arenberger P, Ruzicka T, Berking C. Confocal laser-scanning capillaroscopy: a novel approach to the analysis of skin capillaries in vivo. Skin Res Technol 2009; 15: 476-81.

50 Pierard GE, Arrese JE, Pierre S et al. [Microscopic diagnosis of onychomycoses]. Ann Dermatol Venereol 1994; 121: 259.

51 Tasic S, Stojanovic S, Poljacki M. [Etiopathogenesis, clinical picture and diagnosis of onychomycoses]. Med Pregl 2001; 54: $45-51$

52 Arrese JE, Quatresooz P, Pierard-Franchimont C, Pierard GE. [Nail histomycology Protean aspects of a human fungal bed]. Ann Dermatol Venereol 2003; 130(12 Pt 2): 1254-9.

53 Mogensen M, Thrane L, Joergensen TM, Andersen PE, Jemec GB. Optical coherence tomography for imaging of skin and skin diseases. Semin Cutan Med Surg 2009; 28 196-202.

54 Welzel J. Optical coherence tomography in dermatology: a review. Skin Res Technol 2001; 7: 1-9.

55 Welzel J, Reinhardt C, Lankenau E, Winter C, Wolff HH. Changes in function and morphology of normal human skin: evaluation using optical coherence tomography. Br J Dermatol 2004; 150: 220-5.

56 Abuzahra F, Spoler F, Forst M et al. Pilot study: optical coherence tomography as a non-invasive diagnostic perspective for real time visualisation of onychomycosis. Mycoses 2010; 53: 334-9.

57 Mogensen M, Thomsen JB, Skovgaard LT, Jemec GB. Nail thickness measurements using optical coherence tomography and 20-MHz ultrasonography. Br J Dermatol 2007; 157: 894-900.

58 Welzel J. [Optical coherence tomography]. Hautarzt 2010; 61: 416-20. 